\title{
The Research on Zhuhai Urban Cooperative Distribution System Based on Planning Demand
}

\author{
Jun TAN \\ Zhuhai College of Jilin University \\ Zhuhai, Guangdong, China \\ gdhkwl@163.com
}

\begin{abstract}
With the rapid development of Guangdong-Hong Kong-Macao Greater Bay Area, the completion and operation of the Hong Kong-Zhuhai-Macao Bridge project, the region has already had the mature conditions for building a core city and a transportation hub on the west coast of the Pearl River. Against this background, studying how to build an intensive and efficient city common distribution system which will provide an important support for the construction project of key international logistics center in South China in Zhuhai City will have important practical significance for Zhuhai's urban economic development.
\end{abstract}

Keywords-Guangdong-HongKong-Macao Greater Bay Area; Sustainable Development; Urban Logistics; Common Distribution

\section{INTRODUCTION}

According to the "Report of 2017 National Economic and Social Development Plan", the national development and Reform Commission will speed up the preparation of planning of the Greater Bay Area. As the core city of the West Bank of the Zhujiang River, Zhuhai has made rapid economic development since 2016. GDP reached 222 billion 637 million yuan in 2016. It increased by $8.5 \%$ on the comparable price, and reached 22 thousand dollars per capita, reaching the high level of income economy in the world. The traffic has made a major breakthrough, the completion of the Hong Kong-ZhuhaiMacao Bridge smoothly completed, Zhuhai has been incorporated into the national high-speed rail network, and the construction of the first phase of the Gaolan Port-Shugang Railway Line was completed. With the establishment of Guangdong Free Trade Zone of Hengqin New Area, the National Demonstration Zone for Independent Innovation of the Pearl River Delta and the Gaolan Port National Economic and Technological Development Zone, implement the strategy of the advanced equipment manufacturing industry belt in the West Bank of the Pearl River and promote the construction of roads and railways in Zhuhai will enable to be further integrated into the transportation integration system of Hong Kong, Macao and the West Bank of the Pearl River Delta.

In order to build a core city on the west bank of the Pearl River and a transportation hub city on the west coast of the Greater Bay Area, Urban distribution is an important economic activity that meets the needs of production and living materials, it is of great significance to study and improve the urban distribution efficiency. This article will base on the empirical analysis of the common distribution of city in Zhuhai, Study on the construction of intensive and efficient of smart city with the operation mode of common distribution system, inspire that traditional services of city transport can also embrace the Internet+Era, the key is how to solve the "last mile" carriage of distribution services of goods, to provide a reference for the rationalization of urban distribution and social economic sustainable development.

\section{BRIEF DESCRIPTION OF COMMON DELIVERY IN CITIES}

Urban logistics takes the city as the service target, and the logistics activities that take place around the needs of the city. Urban logistics has narrow and broad understanding: the broad understanding of the concept of urban logistics includes the internal logistics activities of the city and the external logistics activities of the city (such as the input and output of logistics in the city); the narrow concept of urban logistics is that the city logistics is only the logistics within the city's internal category, and the city's logistics of input and output are understood that occurs between the city and the region, which belongs to the category of regional logistics.

Urban logistics is an important basis for the existence and development of modern cities, and occupies an important position in the development of urban economy. However, urban logistics also has obvious external diseconomy to the society and the environment. It is one of the major causes of environmental and social problems that threaten the lives of residents. Urban distribution is an important part of urban logistics. It is one of the core issues in the field of sustainable development of urban logistics to explore how to rationalize and optimize urban distribution to reduce the impact on urban society and the environment.

The common distribution pilot was initiated by the Ministry of Commerce in response to the problems existing in China's cities, such as the difficulty of commodity distribution trucks entering the city, low level of organization, high distribution costs, and low distribution efficiency, among which the Notice of the general office of the Ministry of Finance and the general office of the Ministry of Commerce on organizing the declaration of urban common distribution pilot projects ([2013] No. 21) proposed that scientific planning and Establishing and perfecting the urban distribution service system are the key.

Key support areas and directions: firstly, the construction of public service platform, the relevant requirements are 
implemented in accordance with the "Construction Specification for Urban Joint Distribution Service Platform". Secondly, logistics distribution center, public distribution center and terminal distribution network that is three level distribution network system. Thirdly, the application of advanced technology to carry out management demonstration such as supply chain, Internet of things, information platform, cold chain distribution, path optimization, integrity certification and so on. The four is the application of standardized equipment, encourage the transformation or lease of standardized warehouse, standardize the van standard distribution vehicles, support the establishment of a tray sharing circulation system, and promote standard coding, belt transport and storage cage transportation. The five is the innovation of distribution mode, encouraging the development of common distribution, chain commercial distribution and electronic commerce distribution in the life of service residents, and highlighting the key distribution of fast consumer goods, fresh food, medicine, household appliances and so on, so as to meet the needs of individualization, diversification and convenience of consumption.

\section{ZHUHAI COMMON DISTRIBUTION SYSTEM BASED ON PLANNING DEMAND}

In recent years, Zhuhai has closely centered on the "Framework Agreement for Closer Cooperation in the Region of Zhujiang River" and strengthened cooperation in urban planning, infrastructure, industrial development, environmental protection, and public services. We will formulate and implement the " The close cooperation planning of the region of Zhujiang River " and focus on promoting the convergence of planning for the three cities, transportation infrastructure, regional ecological green network, renovation of rivers systems, and the integration of key urban and rural areas.

With the rapid development of the transportation infrastructure construction in Zhuhai, the comprehensive transportation pattern has basically taken shape, the total transport economy has continued to grow, and the public security capacity has been significantly enhanced. Zhuhai traffic and Transportation Bureau stands at the high level of economic development in the city and proposes to integrate the characteristics of the three major industries closely in Zhuhai, further integrate transportation resources, optimize the transport structure, speed up the cultivation of transport economy, increase the efficiency of social operation, reduce the economic operating costs, and serve the development of economic and social community.

With the development of urban economy, in order to meet the demand of consumers with "diversity and individualization", manufacturers have adopted the production methods with "multi-variety, low-volume", and correspondingly high-frequency, low-volume urban logistics service methods have also emerged. It will inevitably lead to an increase in the total traffic volume of society and highfrequency freight vehicles entering and leaving the center area of cities. This will eventually lead to uneconomical externalities of many urban logistics, such as urban traffic jams, aggravation of urban traffic congestion, and continuous deterioration of urban air quality. The energy consumption is serious and the cost of living space for urban residents is increasing day by day.

Through the extensive application of modern logistics technology, integration of Zhuhai logistics resources, innovation of urban common distribution model, leading products, from warehousing to distribution, IT technology and logistics finance, it provides one-stop urban distribution service for the planned demand of enterprise and customers, building a warehouse allocation based on planned demand. Zhuhai urban distribution system, such as city express, merchant super store distribution, cold chain logistics and logistics finance, has a positive effect on improving the mileage utilization rate of urban freight vehicles, reducing vehicle driving and urban traffic congestion, reducing exhaust emissions and promoting the development of green logistics in cities.

\section{A. Warehousing and distribution integration}

Warehousing and distribution is a combination of warehouses and distribution, not only warehouse network but also distribution network. Customers can choose to enter the nearest warehouse to reduce the logistics cost. The urban distribution network can provide customers with visual monitoring of the whole distribution process, analysis reports of various warehouses and it can help customs analyze at the logistics terminal. So it can guarantee the timeliness. The provision of standardized warehousing and distribution services includes collection, storage, sorting, operation, packing, allocation, distribution, and order performance, which fully embodies the service concept and core value of "common warehousing and joint distribution".

\section{B. City express}

The trunk logistics company provides the collection and delivery of goods, the electronic commerce distribution in the same city and the distribution of small pieces of goods in the city. And provide B2B distribution (business to business) and $\mathrm{B} 2 \mathrm{C}$ retail (business to person) door to door distribution service, the collection of small and medium-sized merchant cargo transportation, to achieve flexible delivery, to meet the needs of real-time loading, unloading and more customers. In different enterprises and different industries to carry out common transportation and joint distribution, improve vehicle full load rate, reduce urban traffic pressure and automobile exhaust emissions, practice the urban sustainable development concept of "energy saving, low carbon".

\section{Distribution of supermarket store}

Multi-store of chain-type hypermarket's distribution service. Through intelligent information systems, with the goal of "controlling operating costs, improving overall distribution efficiency, and comprehensively improving the quality of logistics distribution", we have the intelligent management of warehouses and the process management of distribution vehicles, and have the ability to design and optimize routes. Customized logistics solutions from capacity-matching models, vehicle volumes, response timeliness and customer experience can ensure the stability of services and improve operational efficiency, and provide additional services such as handling, 
loading and unloading, personnel back tracking, documents recycling and sorting of goods.

\section{Cold chain logistics}

Through the integration of socialized cold chain logistics resources, we provide one-stop fresh refrigerated distribution services for fresh agriculture, fresh electricity suppliers, catering enterprises and so on. And we can deliver the goods according to the time of receiving, the temperature of the goods, the way of packing, the standard of the label and so on, so as to ensure the accuracy and the rate of delivery, and ensure the quality of the fresh products.

\section{E. Logistics finance}

Through financial services and Internet technology, it can reduce costs and increase the efficiency for customers. Freight loans, car loans and car insurance and other products, with making loan more convenient and rapid, solve the problem of financing difficult for small and medium-sized enterprises and help the customers to expand their scale. Freight loans can meet the needs of the daily flow funds of small and medium enterprises, help the logistics enterprises and owners to slow down the pressure of the freight funds and support the prepayment of freight. It will reduce the cost of purchasing power and help the small and micro logistics enterprises that develop the business and growth the scale.

\section{URBAN DISTRIBUTION INFORMATION PLATFORM BASED ON LBS}

Establishing an urban distribution vehicle information service $\mathrm{O} 2 \mathrm{O}$ (online to offline) platform through LBS (location service) technology, is an effective means to solve the problems of low level of organization, high distribution costs, and low distribution efficiency. Due to the incomplete informationization, lack of standardization, and low efficiency of services in the traditional freight market, LBS technology based on mobile Internet can not only obtain driver's location information, but also can obtain information on empty trucks It can reduce the driver's waiting time, empty distance, reduce the driver's waiting time and empty distance, reduce the driver's waiting time, increase the full load rate, and improve the efficiency of logistics information transmission., improve the utilization of transport vehicles.

By downloading APP application of city distribution, information platform connects drivers and users directly. Integrate the real-time position information of idle wagon, release the real-time freight demand of the owner, let the freight car talk with the owner directly, reduce the middle link, solve the problem that it is difficult and expensive to find a car. After entering the start and stop locations, type of car, numbers of people and other requirements, the platform will give the corresponding price, and the user will be able to "confirm the car" ( Figure 1).
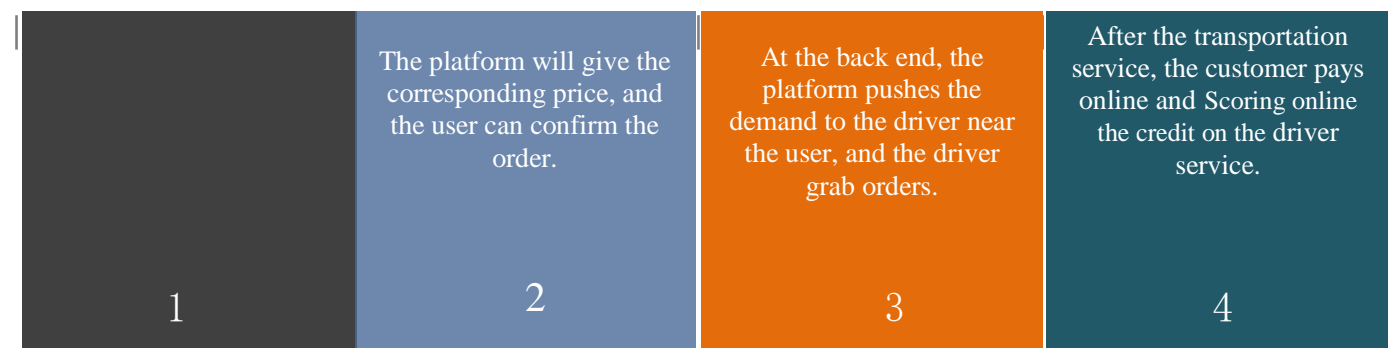

Fig. 1. "Car process" in Client app

Based on the MCT, mobile phone, using mobile Internet technology to develop the application entrance of APP and WeChat, build a communication bridge between logistics company, cargo owner and driver, ensuring that information is delivered and updated in time and effectively. The two sides of the transaction can either place their own transactions online, or choose free secured transactions on the platform. The platform avoids the occurrence of breach of contract between two parties through the margin system.

Based on station positioning and GPS technology of mobile phone, monitoring of carrier vehicles, which achieves transparent transportation management, and provides LBS services. At the same time, the real name authentication is carried out on both sides to ensure the authenticity of the transaction subject, and the credit evaluation system guides the customers to abide by the trading rules. Based on the analysis of freight transaction data, we provide freight forwarding service for creditworthy users. Online cargo insurance supports purchase immediately online and settle a claim, reducing transport risks effectively. Form a distribution model based on 020 of LBS.
The platform also provides services as the third party agente, that is, the agente will integrate the transportation information and form an efficient match according to the owner's condition. The vehicle information management system ensures the visualization of the transportation information. The transparent price system makes the enterprise reduce the logistics cost greatly of the enterprise in the case of paying the reasonable commission and the freight cost. We will rebuild the new order of logistics industry through continuous optimized the services.

Logistics companies can also release information on goods and dispatch their vehicles online to determine the freight. Apply for a dedicated line, accept shipper's online order, expand collection business online. Supervise the implementation of bilateral transactions through the margin system, and ensure the safety of cargo transportation by cargo insurance online and location tracking technology.

For the security issues, the most important thing in logistics industry, in addition to the three certificates of vehicle owners, the system also implements the user's real-name system. The 
information platform makes a return visit to all registered users, and determines the true and reliable information provided by the information platform, which greatly avoids the false information problems of some large logistics websites. Secondly, the information platform builds independent credit evaluation system for owners and shippers, and the vehicle ban for lifetime when it breach of contract. At the same time, the information platform also uses mobile phone base station positioning and GPS positioning technology to ensure the realtime monitoring of the vehicle which ensure the safety of vehicles and goods, freight guarantee online, vehicle security or other measures.

\section{REFERENCES}

[1] D.C.Fang, L.Y. Liu, University Delivery Controls Torture Campus Governance, Education Weekly, 2016.

[2] Y. Zhao, The current status of college students' takeaway consumption and analysis of its influencing factors, Modern Business, 2017.

[3] M.Y. Wang, Z.X. Zhao, Research Report of Market on University Takeaway O2O, Logistics Technology, 2016.

[4] Y.F. Liang, Research on School Logistics Model of Outsourcing Public Crowd, Value Engineering, 2017.

[5] C. Deng, Thoughts on logistics distribution of college catering O2O companies, Modern Business, 2017. 